\title{
An indigenous cluster beam apparatus with a reflectron time-of-flight mass spectrometer
}

\author{
G RAINA $^{\mathrm{a}}$, G U KULKARNI ${ }^{\mathrm{a}}$, R T YADAV ${ }^{\mathrm{a}}$, V S RAMAMURTHY ${ }^{\mathrm{b}}$ and \\ C N R RAO ${ }^{\mathrm{a}, *}$ \\ ${ }^{\mathrm{a} C h e m i s t r y}$ and Physics of Materials Unit, Jawaharlal Nehru Centre for \\ Advanced Scientific Research, Jakkur PO, Bangalore 560 064, India \\ ${ }^{\mathrm{b}}$ Department of Science and Technology, Technology Bhavan, New \\ Mehrauli Road, New Delhi 110 016, India
}

MS received 14 February 2000

\begin{abstract}
The design and fabrication of a Smalley-type cluster source in combination with a reflectron based time-of-flight (TOF) mass spectrometer are reported. The generation of clusters is based on supersonic jet expansion of the sampling plume. Sample cells for both liquid and solid targets developed for this purpose are described. Two pulsed Nd-YAG lasers are used in tandem, one $(532 \mathrm{~nm})$ for target vapourization and the other $(355 \mathrm{~nm})$ for cluster ionization. Methanol clusters of nuclearity up to 14 (mass $500 \mathrm{amu}$ ) were produced from liquid methanol as the test sample. The clusters were detected with a mass resolution of $\sim 2500$ in the R-TOF geometry. Carbon clusters up to a nuclearity of 28 were obtained using a polyimide target. The utility of the instrument is demonstrated by carrying out experiments to generate mixed clusters from alcohol mixtures.
\end{abstract}

Keywords. Mass spectrometer; cluster source; instrumentation.

\section{Introduction}

Clusters constitute an intermediate state between bulk and atomic/molecular species of substances. The evolution of structure, reactivity and electronic properties with increasing cluster size forms a topic of great interest. Clusters of metals and semiconductors have been studied in the form colloids ${ }^{1}$, as adsorbed species on substrates in vacuum ${ }^{2}$ as well as in molecular beams ${ }^{3}$. Cluster generation in the vapour phase is particularly advantageous in that clusters of any substance may be obtained devoid of surfactants or capping agents. The presence of clusters in molecular beams was first reported by Ramsey ${ }^{4}$ as early as in 1948 . Bentley ${ }^{5}$ and Henkes ${ }^{6}$ independently detected jet-generated clusters of carbon dioxide by using mass spectrometers. Since 1970, experimental work on clusters has progressed significantly due to the advent of lasers, supersonic pulsed valves and improvised mass spectrometers. Thus, Smalley and coworkers ${ }^{7}$ have reported the production of $\mathrm{Ni}_{n}, \mathrm{Fe}_{n}, \mathrm{Cr}_{n}, \mathrm{~W}_{n}$, and $\mathrm{Mo}_{n}$ clusters using a pulsed laser vapourization source. Their work culminated in the discovery of the fullerenes ${ }^{8}$, which has given much impetus to the field of cluster science.

\footnotetext{
*For correspondence
} 
Recent literature on cluster beams includes studies on the stability of Met-Cars ${ }^{9}$, reactivity of organometallic complexes ${ }^{10}$, metastable cluster ions such as ammonia, methanol and xenon ${ }^{11}$. Infrared spectroscopic measurements have been carried out on mass selected clusters of organic molecules ${ }^{12,13}$, while metal ${ }^{14,15}$ and semiconductor ${ }^{16-18}$ clusters have been examined by photoelectron spectroscopy. Clusters of iron and its oxide have been investigated for magnetic properties ${ }^{19}$. Harfenist et $a l^{20}$ obtained a quantum dot lattice of mass selected Ag clusters capped with self-assembling thiol spacers.

The essential components of a cluster beam apparatus are a cluster source and a mass spectrometer. Simple sources such as the Knudsen cell ${ }^{21}$ or an inert gas condensation source $^{22}$ have been used extensively in the past. More recently, inert gas pulsed valves operating in the range of few microseconds are being used to realize supersonic jet beams. Such beams are known to produce good cluster yields besides lowering the internal energy down to a few kelvins ${ }^{7}$. The clusters produced in a molecular beam are mostly neutral and have to be ionized in the mass spectrometer. Ions of different massto-charge ratios are separated by electrostatic deflection, magnetic deflection or using a combination of the two. Quadrupole mass spectrometers on the other hand are based on selective response of ions to RF and DC electric fields. Time-of-flight mass spectrometers segregate ions according to their velocities and are ideally suited for cluster studies owing to their high resolution, selectivity and mass range.

We considered it most worthwhile to initiate research on cluster beams and therefore have undertaken the design-development and fabrication of a pulsed laser vapourization cluster source coupled to a reflectron time-of-flight mass spectrometer. Such an integrated system is, however, not commercially available. We have therefore fabricated most of the parts indigenously. The instrument is now operational with the mass spectrometer tuned to a resolution of $\sim 2500$. Clusters from both liquid and solid targets have been generated using different types of sample cells and were successfully detected. Clusters of ethanol, methanol and their mixtures of varying compositions have been subjected to mass analysis. A variety of hybrid clusters were obtained from the vapour mixtures. Carbon clusters have been produced using graphite or polymer based targets. The equipment can be used effectively for cluster production of a wide variety of materials.

\section{Instrumentation}

Figure 1 shows the system with two vacuum chambers separated by a gate valve and a flight-tube attached to the second chamber. The two chambers were designed for cluster generation and mass detection respectively. An engineering drawing of the system was prepared using AutoCAD software. The vacuum chambers were locally fabricated (New Poona Industries, Pune). The chambers are connected through knife-edge flanges using copper gaskets. The first chamber is pumped by an oil diffusion pump (1200 1/s) attached to a liquid nitrogen trap while the second chamber along with the flight-tube is connected to a turbomolecular pump (Alcatel, France) with a pumping speed of $900 \mathrm{l} / \mathrm{s}$. The diffusion and the turbomolecular pumps are backed by separate rotary pumps. A magnetron and an ion-gauge (Edwards, UK) are employed to read vacuum in the first and the second chamber, respectively. After baking, a vacuum of $\sim 10^{-9}$ torr is obtained routinely in both the chambers.

The various internal components were fabricated with optimal geometry so as to fit into the different parts of the vacuum chambers. An extraction-acceleration grid assembly, a focussing assembly, a reflectron set-up and a detector form the essential components of 


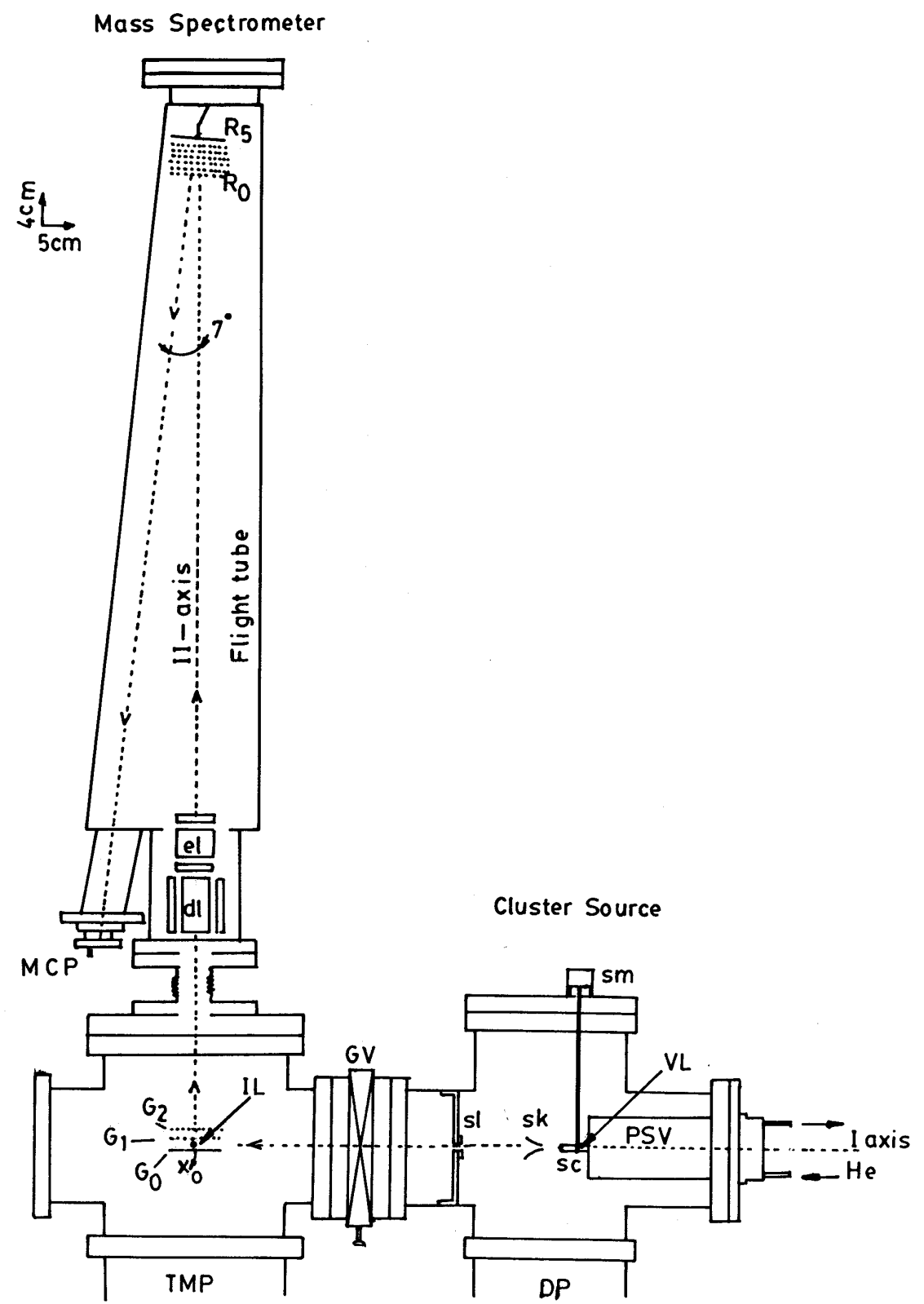

Figure 1. A schematic drawing of the cluster source attached to the time-of-flight mass spectrometer: PSV; pulsed supersonic valve, MCP; microchannel plate detector, sc; sample cell, sm; stepper motor, sk; skimmer, sl; slit, dl; deflection plates, el; einzel lens, $x_{0}$; point of ionization, VL; vapourization laser, IL; ionization laser, $G_{0}, G_{1}$ and $G_{2}$; the repeller, extractor and accelerator grids, $R_{0}-R_{5} ;$ reflectron grids, D.P.; diffusion pump, TMP; turbomolecular pump and GV; gate valve. 
the mass spectrometer. The extraction-acceleration grids, $G_{1}$ and $G_{2}$, are made up of Ni mesh (diameter $6.4 \mathrm{~cm}$, grid 40 wires $/ \mathrm{cm}$, transmission $\sim 85 \%$ ) laser-welded to circular steel rings of $9.2 \mathrm{~cm}$ diameter. The grids along with the repeller plate, $G_{0}$, are mounted parallel on a steel frame at a desired spacing ${ }^{23}$ using macor bushes. A spacing of $1.44 \mathrm{~cm}$ between the grids was chosen so that the ratio of the diameter and the spacing was $\sim 4$. The grid assembly is then mounted horizontally in the second chamber with the mid-point of $G_{0}$ and $G_{1}$ coinciding with the centre of the chamber which is also the focal point of the ionization laser (figure 1). Electrical connections are provided using vacuum feedthroughs. The focussing assembly consists of two pairs of rectangular deflection plates $\left(3.77 \times 7.99 \mathrm{~cm}^{2}\right)$ and an einzel lens (diameter $\left.4 \mathrm{~cm}\right)$, all made of stainless steel. These are rigidly fixed to a steel frame using macor spacers. The assembly is placed at the entrance of the flight tube. The reflectron consists of a set of parallel grids, $R_{0}$ to $R_{5}$, similar to the ones used in the ionization region. The grids are mounted at the end of the flight tube such that the normal at the centre of the grids subtends an angle of $3.5^{\circ}$ to the II axis of the flight-tube. A microchannel plate detector of $1.8 \mathrm{~cm}$ diameter (R M Jordan, USA) is placed symmetrically at the other end of the flight tube.

Several variations have been tried out for cluster generation. Routinely, a sample cell in combination with a pulsed supersonic valve (R M Jordan, USA) is placed in the first chamber behind a slit-skimmer assembly. The latter consists of an adjustable slit mounted on a stainless steel circular plate carrying an O-ring in order to facilitate differential pumping of the two chambers. The skimmer is made of nickel with a $1.5 \mathrm{~mm}$ orifice (Beam Dynamics, USA). Liquid samples were either fed directly into the pulsed valve, mixed with pressurized helium, or dropped in tiny amounts in front of the valve in vacuum under additional pumping. For solid targets, a sample cell based on the design by Smalley and coworkers ${ }^{24}$ has been developed. It has a $0.5 \mathrm{~mm}$ opening mating with the nozzle of the pulsed valve as shown in figure 2 . The opening further leads to an inner cavity of $1 \mathrm{~mm}$ diameter which runs for a length of $12 \mathrm{~mm}$ before widening to a $3 \mathrm{~mm}$ bore of $25 \mathrm{~mm}$ length. The cavity is intercepted perpendicularly at the junction by a semi-cylindrical slot of $3 \mathrm{~mm}$ diameter for the target rod (see figure 2). There is also an opening $(1 \mathrm{~mm})$ opposite the slot which allows in the vapourization laser to be focussed on the target. All the openings on the cell have been lined with teflon for better sealing. The target is made to rotate and translate simultaneously using a mechanism driven by a stepper motor through a vacuum feedthrough.

Pulsed Nd-YAG lasers from Spectra Physics, USA (pulse width 5-7 ns and frequency $10 \mathrm{~Hz}$ ) have been used in the Q-switch mode for ablation and ionizaiton. The $532 \mathrm{~nm}$ output of the vapourization laser operating at $\sim 40 \mathrm{~mJ} /$ pulse is focussed onto a $1 \mathrm{~mm}$ spot on the target rod using a lens of $50 \mathrm{~cm}$ focus mounted on an $x-y-z$ manipulator. For ionization, the $355 \mathrm{~nm}$ output from the second laser $(80 \mathrm{~mJ} /$ pulse $)$ is used.

\section{System integration and calibration}

The vacuum system was first assembled on a metal frame and fittings such as the ion gauge head and the gate valve were tested for vacuum leak using a residual gas analyzer. The slitskimmer assembly in the first chamber was aligned along the I-axis of the system (figure 1) using a He-Ne laser. The pulsed valve placed along the axis was operated at $10 \mathrm{~Hz}$ backed with 10 atmosphere pressure of a methanol-helium mixture. The mixture was produced by passing helium (99.999\% pure) through a sample cell containing methanol (HPLC grade, Aldrich). The methanol beam could be detected by the residual 


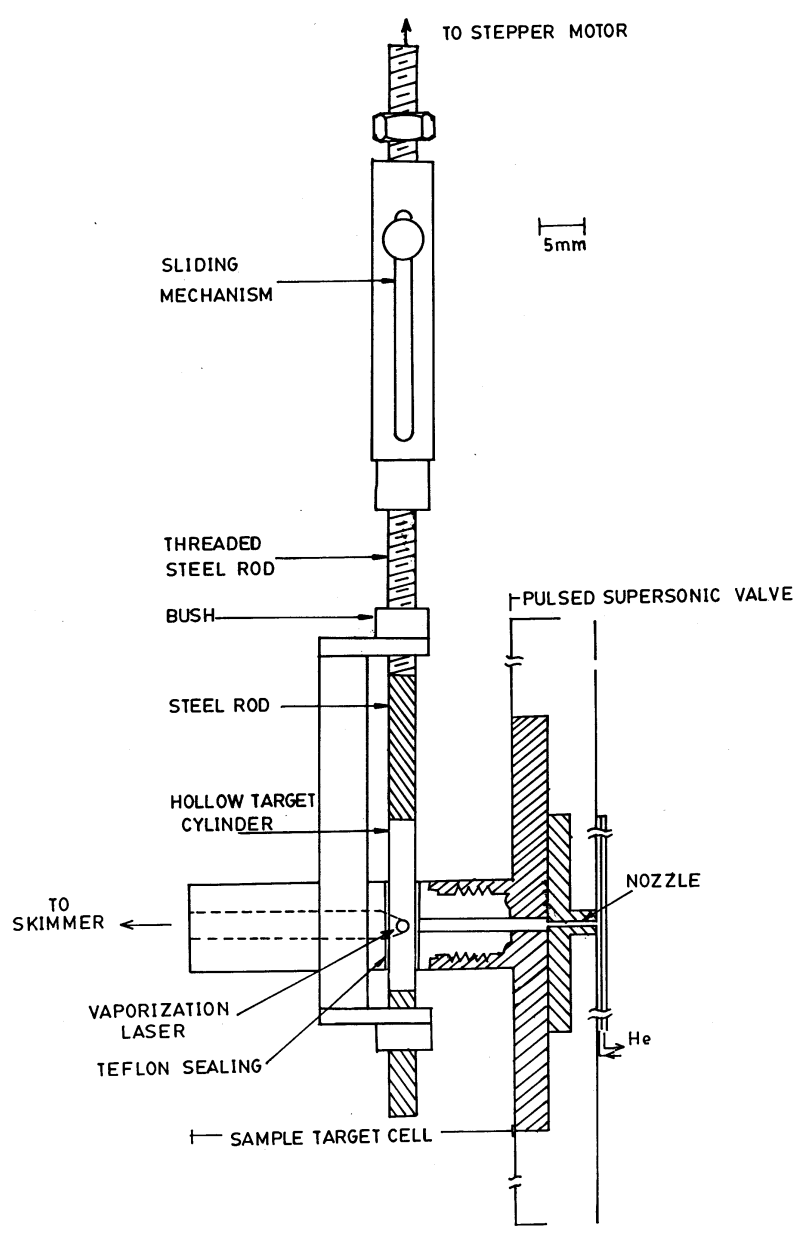

Figure 2. Schematic drawing of the solid cluster source. The sample target cut in the form of a cylinder is held pressed against the cell using a clamp. The vapourization laser produces a spiral groove by ablating the sample material as the target rotates and translates.

gas analyzer placed in the ionization chamber along the same axis. The spatial alignment of the molecular beam with the ionization laser was done by introducing a steel rod in vacuum which carried a perpendicular bore of $1 \mathrm{~mm}$, close to its tip. The rod was held perpendicular to the molecular beam such that the beam could pass through the bore. In this position, the laser was focussed at the tip of the rod.

The extraction-acceleration grid voltages were calculated using the first order focusing condition ${ }^{3}$,

$$
\partial T / \partial x_{0} \rightarrow 0
$$


here, $T$ is the time-of-flight of the ion and $x_{0}$ is the point of ionization between $G_{0}$ and $G_{1}$ (figure 1). For $d_{1}=d_{2}=1.44 \mathrm{~cm}$ and $L=1.54 \mathrm{~m}$ and $x_{0}=d_{1} / 2=0.72 \mathrm{~cm}$, voltage values of 3000 and $1200 \mathrm{~V}$ were obtained for $G_{0}$ and $G_{1}$ respectively. The einzel lens in the focussing assembly was given $1275 \mathrm{~V}$ with the deflection plates held at small voltages of $\sim 50 \mathrm{~V}$.

The microchannel plate detector was initially mounted at the end of the flight tube and the spectrometer was operated in the linear mode. The pulsed valve and the ionization Q-switch were triggered externally at $10 \mathrm{~Hz}$ using a digital delay generator (EG\&G, USA) as shown in figure 3. The output from the detector was fed to a fast timing preamplifier and then on to a multi-channel scaler (EG\&G, USA) connected to a personal computer for storage of data. Mass of a cluster ion was calculated using the equation ${ }^{3}, T=c \sqrt{ } m$. Here $T$ is the time-of-flight of the ion, $m$ is the mass of the ion and $c$ is a constant given by the equation,

$$
\begin{aligned}
c=(1 / z)^{1 / 2}\left[\sqrt{ }\left(2 * x_{0} / E_{1}\right)\right. & +\sqrt{2} / E_{2}\left(\left\{E_{1} * x_{0}+E_{2} * d_{2}\right\}^{1 / 2}-\left(E_{1} * x_{0}\right)^{1 / 2}\right) \\
& +L /\left(\sqrt{2}\left(E_{1} * x_{0}+E_{2} * d_{2}\right)^{1 / 2}\right],
\end{aligned}
$$

where $z$ is the charge on the ion and $E_{1}$ and $E_{2}$ are the electric fields in the extraction and acceleration region, respectively. A value of $c=2.667 \mathrm{~ms}^{\mathrm{amu}} \mathrm{am}^{-1 / 2}$ was obtained from the experiment with 3000 and $1200 \mathrm{~V}$ applied on $G_{0}$ and $G_{1}$, respectively.

\section{CONTROL ELECTRONICS}

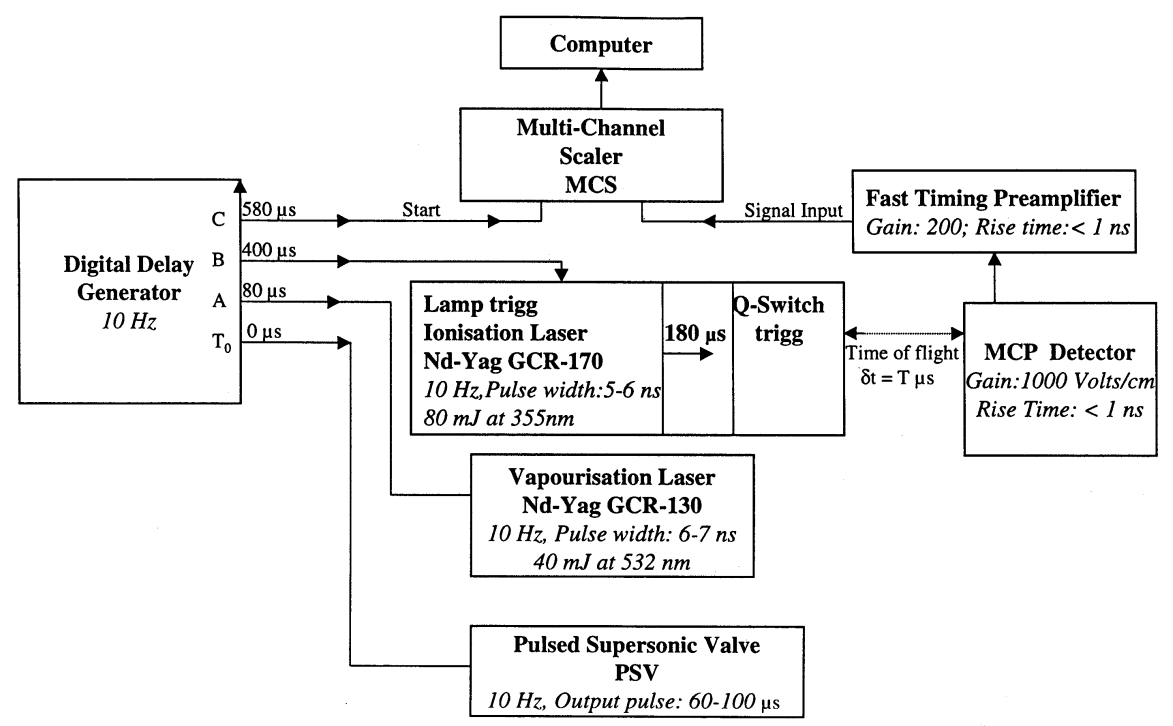

Figure 3. Schematic drawing of the control electronics. Digital delay generator operating at $10 \mathrm{~Hz}$ triggers the pulsed supersonic valve with $T_{0}$ output. The flashlamps of the vapourization and the ionization lasers and the start of the multichannel scaler are all triggered by the delay generator with delay pulses with respect to $T_{0}$, of $\mathrm{A}=80, \mathrm{~B}=400$ and $\mathrm{C}=580 \mathrm{~m}$, respectively. The microchannel plate detector output is amplified by a fast timing preamplifier, which is fed to the discriminator of the scalar. The data is stored and analyzed using a pentium computer. 
Temporal synchronization of the ionization laser pulse with the molecular beam pulse (width $\sim 100 \mathrm{~ms}$ ) was achieved for a delay of $\sim 400 \mathrm{~ns}$. The beam velocity was indeed supersonic $\left(\sim 1.2 ¥ 10^{3} \mathrm{~m} / \mathrm{s}\right)$. After obtaining the signal in the linear mode, the reflectron grid assembly was introduced. A parabolic voltage pattern was applied to the reflectron grid assembly with $R_{0}$ held at ground potential, $V=a x^{2}$ where $a=148.5 \mathrm{~V} / \mathrm{cm}^{2}$ and $x$ is the distance of a grid from $R_{0}$. Accordingly, voltages of 131, 524, 1181, 2100 and $3280 \mathrm{~V}$

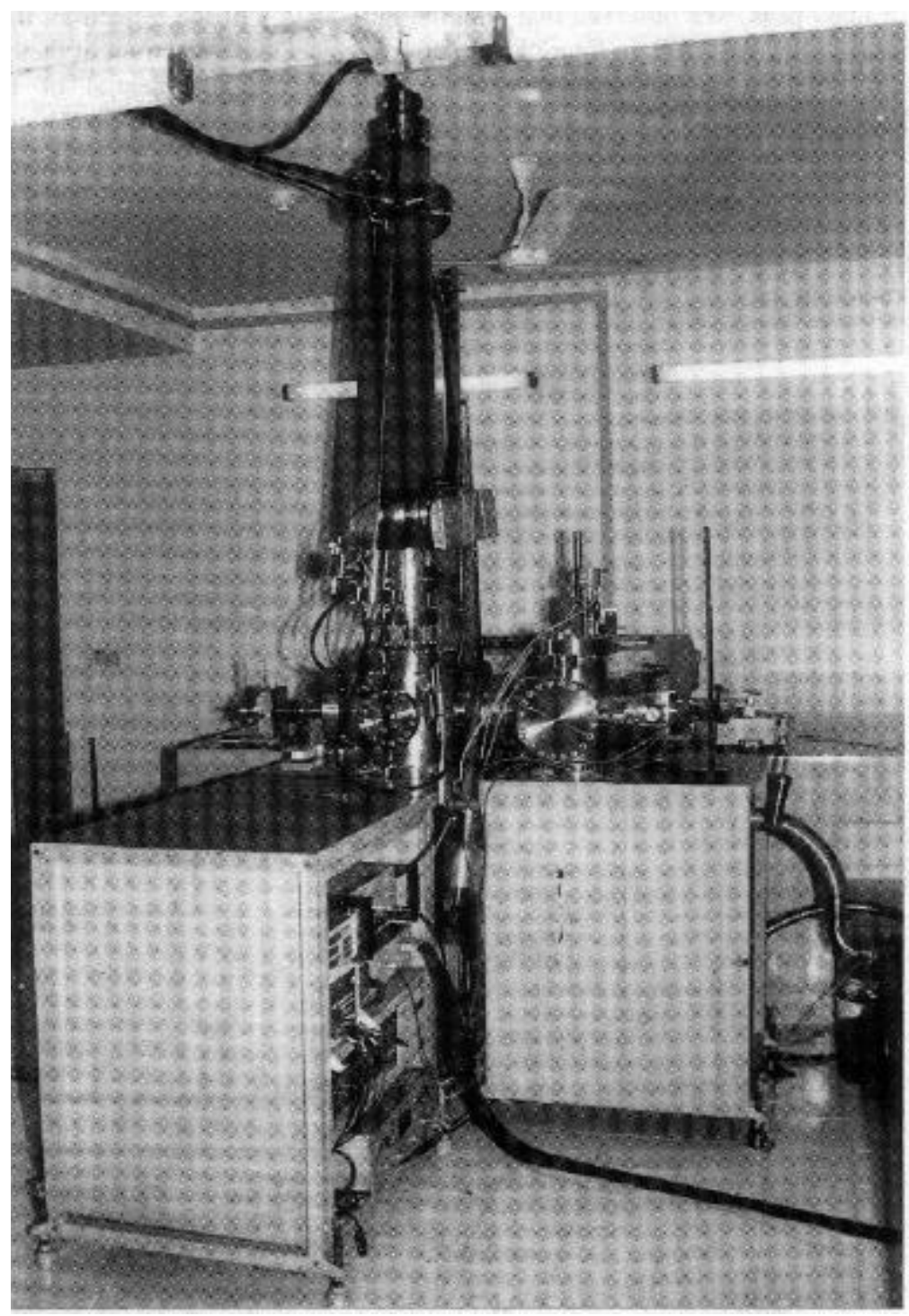

Figure 4. A photograph of the fully assembled cluster apparatus with the R-TOF mass spectrometer. 
were given to the grids $R_{1}$ to $R_{5}$, respectively. The retarding field in the reflectron narrows down the velocity distribution in an ion packet and deflects it towards the detector. In figure 4 we show a photograph of the fully assembled apparatus.

\section{Performance}

The TOF mass spectrometer operating in the linear mode was able to detect methanol clusters with a resolution of $R=T / 2 \Delta T \sim 800$, where $\Delta T$ is the full-width half maximum of a mass peak. We observed that with the introduction of the reflectron, the resolution improved beyond 2500. In figure 5, we show a typical mass spectrum of methanol cluster beam obtained in the reflectron mode. The ionization potential of methanol is $10.85 \pm 0.01 \mathrm{eV}$, slightly higher than the three photon energy at $355 \mathrm{~nm}$. Therefore, we do not observe the methanol monomer (32 amu) in the spectrum. The dimer (64 amu) and the higher nuclearity clusters $(96,128,160 \mathrm{amu}$ etc.) corresponding to potential drops of $\sim 0.2$ to $0.3 \mathrm{eV}$ can be seen since three photon ionization becomes possible ${ }^{25}$. The spectrum in figure 5 shows protonated methanol cluster ions up to a nuclearity of 14 with populations tapering off around $n=7$. The inset shows a full-width half maximum of

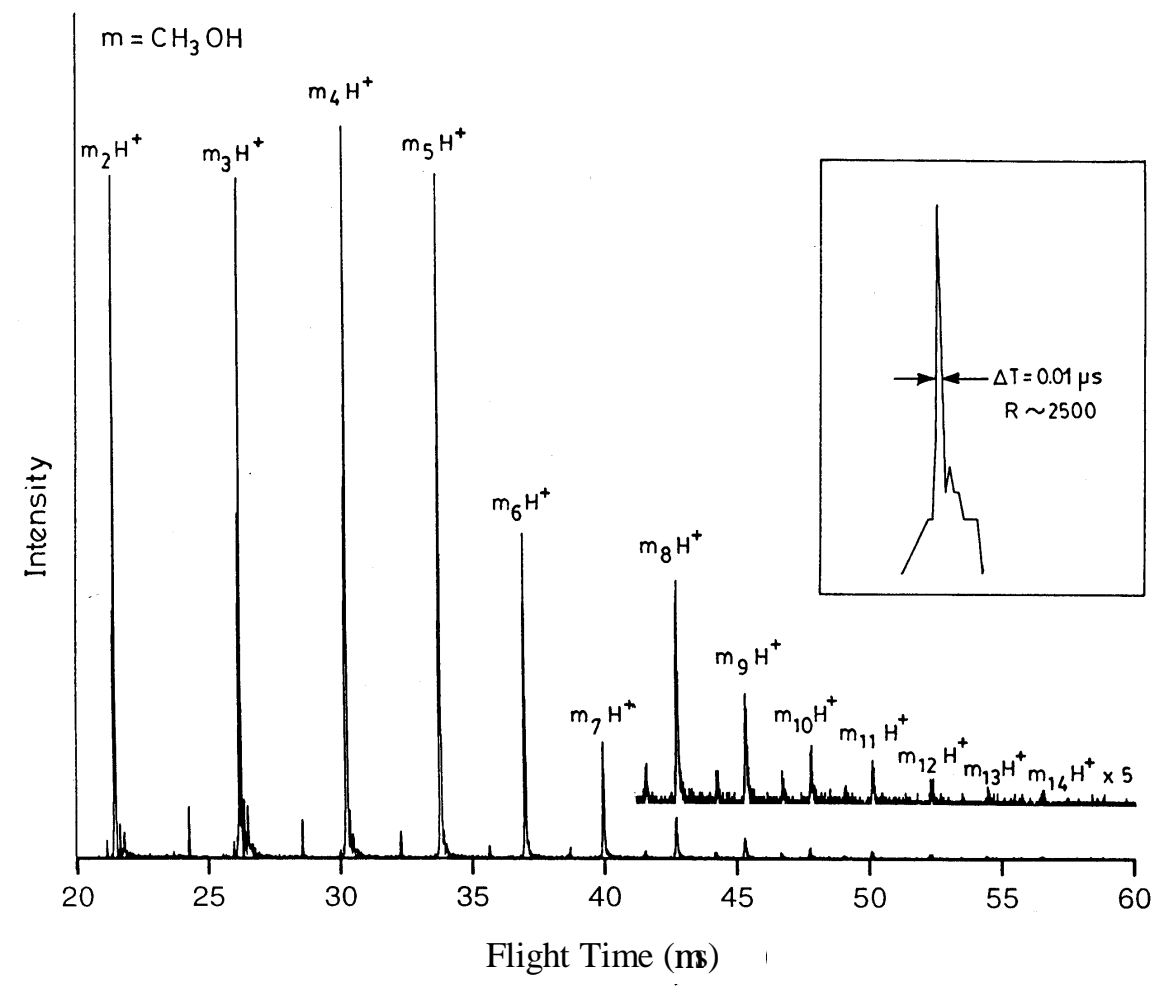

Figure 5. Time-of-flight mass spectrum of methanol clusters obtained with our RTOF mass spectrometer. The inset depicts typical resolution of the spectrometer. In addition to the $\left(\mathrm{CH}_{3} \mathrm{OH}\right)_{n} \mathrm{H}^{+}$peaks, small intermediate peaks corresponding to $\left(\mathrm{CH}_{3} \mathrm{OH}\right)_{n}\left(\mathrm{H}_{2} \mathrm{O}\right) \mathrm{H}^{+}$are also observed. 
$0.01 \mathrm{~m}$ for the mass peak corresponding to the $\left(\mathrm{CH}_{3} \mathrm{OH}\right)_{11} \mathrm{H}^{+}$cluster. The protonated methanol peaks are believed to arise due to a rapid intracluster proton-transfer reaction following ionization of the neutral clusters ${ }^{26}$. From figure 5, we also see mass peaks (83, $115 \mathrm{amu}$ etc.) due to methanol clusters attached to a water molecule. It is interesting that water present as an impurity in extremely small amounts $(\sim 10 \mathrm{ppm})$ gives rise to such mixed clusters. Methanol subjected to dry distillation, however, did not produce mixed clusters.

By using the solid sample cell, carbon clusters were obtained from a graphite rod. The pulsed valve was operated with helium at $10 \mathrm{~atm}$ and the vapourization laser pulse was delayed with respect to the helium pulse by $80 \mathrm{~ms}$. The graphite target was rotated at $\sim 2$ rpm. We were able to observe carbon clusters of nuclearity 20 (240 amu). Higher mass clusters can be obtained by fine tuning of laser fluence to prevent multiphoton fragmentation ${ }^{27}$.

We have employed the TOF mass spectrometer to characterize the ablation products. For these experiments, the sample is placed in the ionization chamber between the repeller $G_{0}$ and extraction $G_{1}$ grids, at a positive voltage with respect to $G_{1}$. The $355 \mathrm{~nm}$ output of Nd-YAG operating at low power is used for simultaneous ablation and

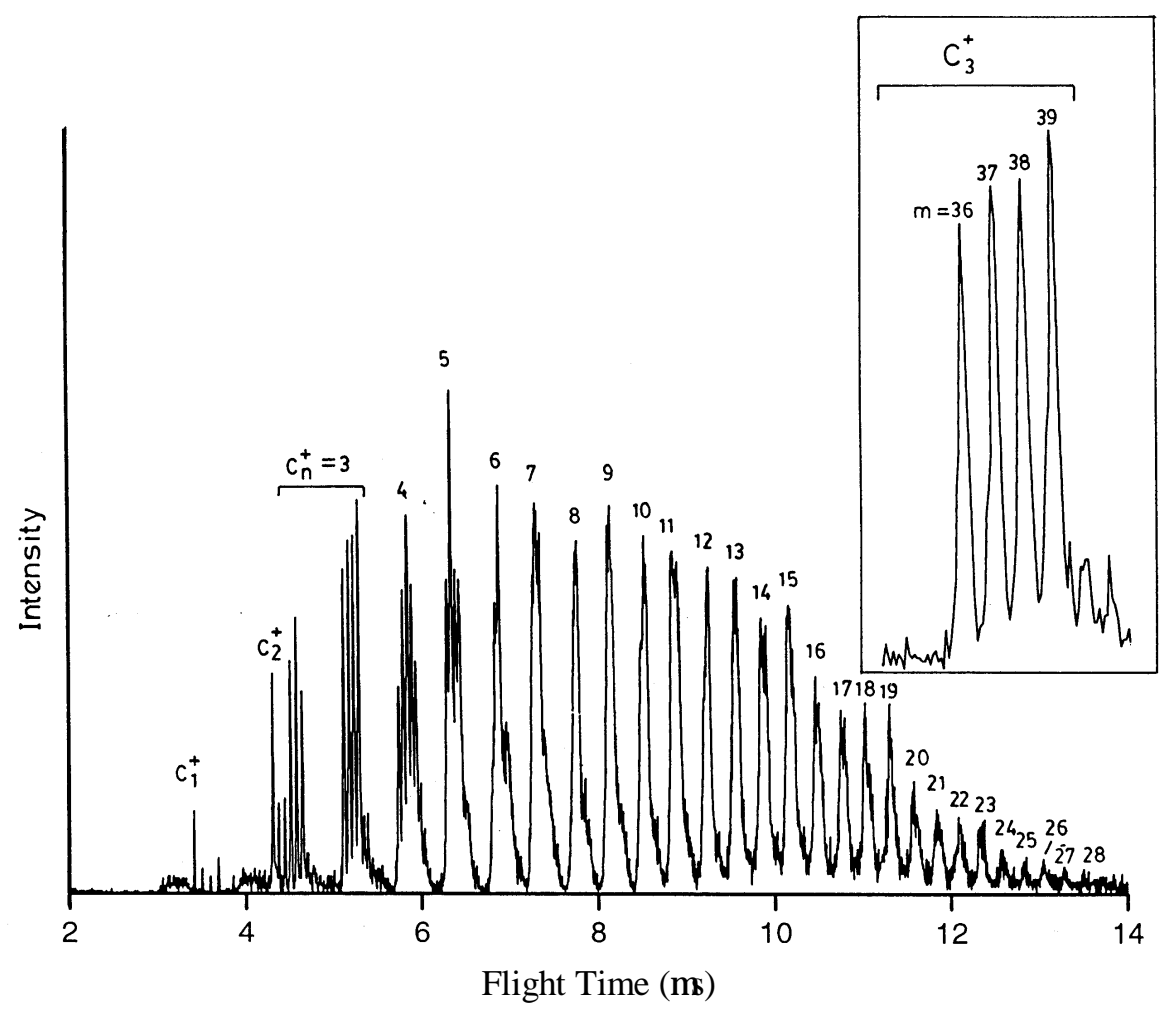

Figure 6. Time-of-flight mass spectrum of a sample of Kapton polyimide subjected to ablation using the $355 \mathrm{~nm}$ laser output. Carbon clusters up to a nuclearity of 28 are detected. The inset shows details of the spectrum in the vicinity of $\mathrm{C}_{3}^{+}$cluster ion. 
ionization. As shown in figure 6, a Kapton polyimide sample under laser ablation gave a mass spectrum rich in carbon clusters ${ }^{28}$. Mass peaks corresponding to carbon clusters of nuclearity from 1 to 28 were observed with the intensity maximum around $\mathrm{C}_{5}{ }^{+}$. The inset in the figure shows well resolved peaks of the trimer at 37, 38, 39 amu. We attribute these peaks to successive proton attachments to the carbon clusters, because of the proton-rich nature of the polyimide. Isotope effect may also contribute to this degeneracy ${ }^{28}$. The mass spectrometer was also tested using $C_{60}$ powder placed in a $0.5 \mathrm{~mm}$ capillary groove which was inclined at $45^{\circ}$ to the laser beam.

\section{Some new results with binary alcohol mixtures}

We have carried out experiments to generate clusters from known mixtures of alcohols. Homogeneous and binary nucleation of molecules has been examined earlier with vapour mixtures under steady flow through supersonic nozzles ${ }^{29}$. Wakisaka et al ${ }^{30}$ carried out mass spectrometry on micro-droplets of mixtures of methanol, water and acetonitrile and studied interactions involved in the nucleation of binary clusters. Our objective was to study how, in the case of alcohol mixtures, the vapour composition can influence hybrid cluster formation. We have analysed the cluster populations of different nuclearities and compositions arising from ethanol-methanol mixtures.

Binary mixtures of ethanol and methanol of different molar compositions were supersonically expanded under similar conditions using $\mathrm{He}$ as the carrier gas. The composition of the vapour was determined using a residual gas analyser placed close to the cluster production unit. The cluster formation was monitored using the time-of-flight mass spectrometer. As an example, we show the clusters obtained with ethanolmethanol vapour of varying compositions (72:28, 49:51, 35:65 and 14:86) in figure 7. We observe single-component as well as mixed clusters up to nuclearities of 4 . Mixed clusters with equal proportions of methanol and ethanol are seen to dominate the spectra. The populations of the mixed clusters were analyzed as a function of the vapour composition (figure 8). Methanol-rich clusters (figure 8a) reach the maximum population at a vapour composition of 35:65 while the ethanol-rich clusters show a peak around a composition of 50:50 (figure $8 \mathrm{c}$ ). The higher nuclearity clusters were generally less abundant in both cases. The dimer (figure 7b) with one molecule of each, reaches the maximum population for a molar composition of 35:65, just as the methanol-rich clusters (figure 8a). This behaviour seems to be related to the difference in the condensation temperatures of the two liquids. Note that the boiling points of methanol and ethanol are 337.8 and $351.4 \mathrm{~K}$ respectively.

\section{Conclusions}

A cluster source apparatus coupled to a reflectron time-of-flight mass spectrometer has been designed and fabricated. Different sample cells attached to pulsed supersonic valve have been developed to generate cluster beams from liquid and solid targets, the latter in conjunction with a vapourization laser. The mass spectrometer with a resolution of $\mathrm{R} \sim 2500$ is capable of detecting masses of several thousand amu. The current focus of work is on the development of sample cells for higher mass clusters as well as enhancing the resolution of the reflectron using SIMION ${ }^{31}$. Using this instrument, clusters of composite materials, hydrogen bonded organic molecules and colloidal preparations are 


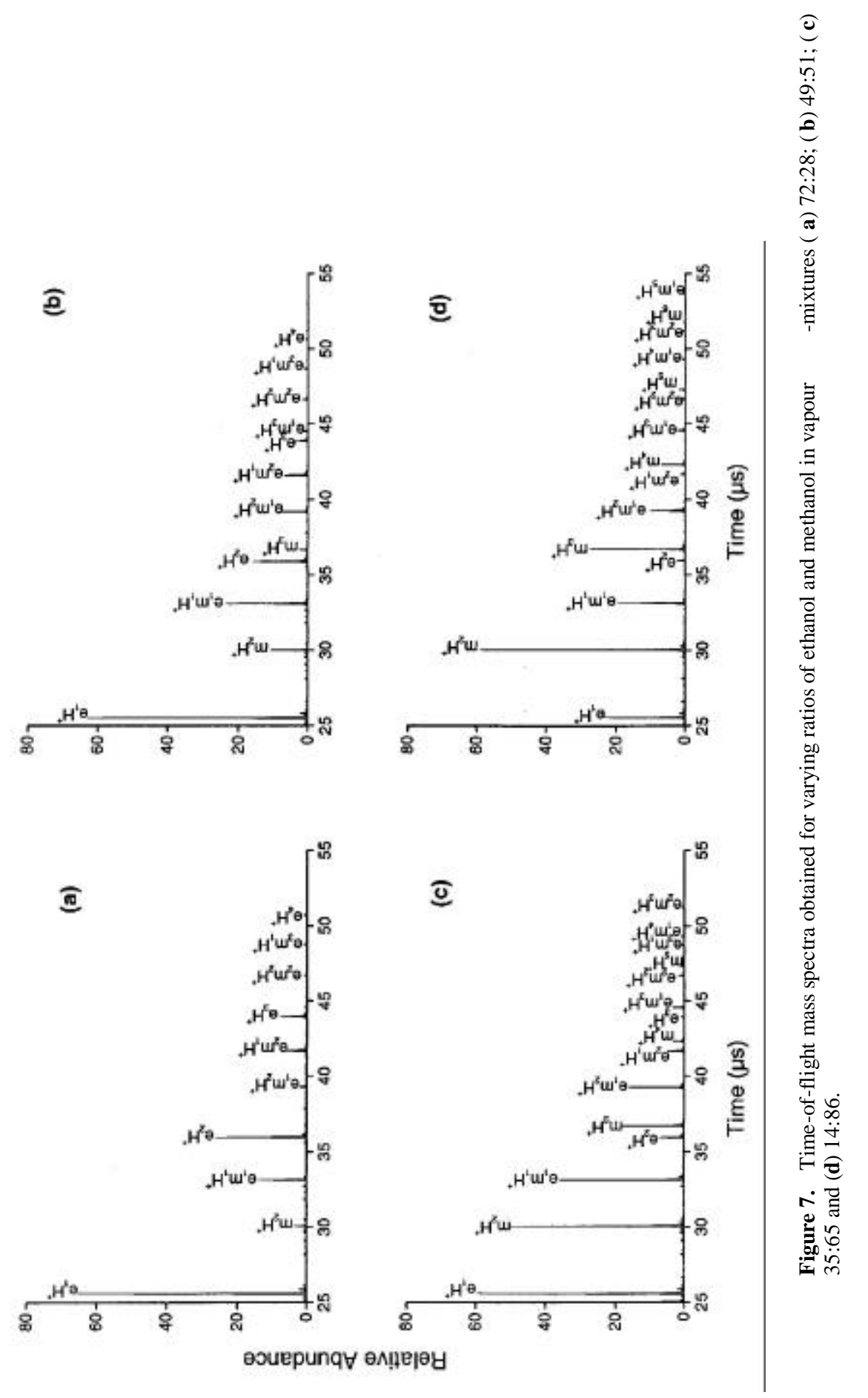



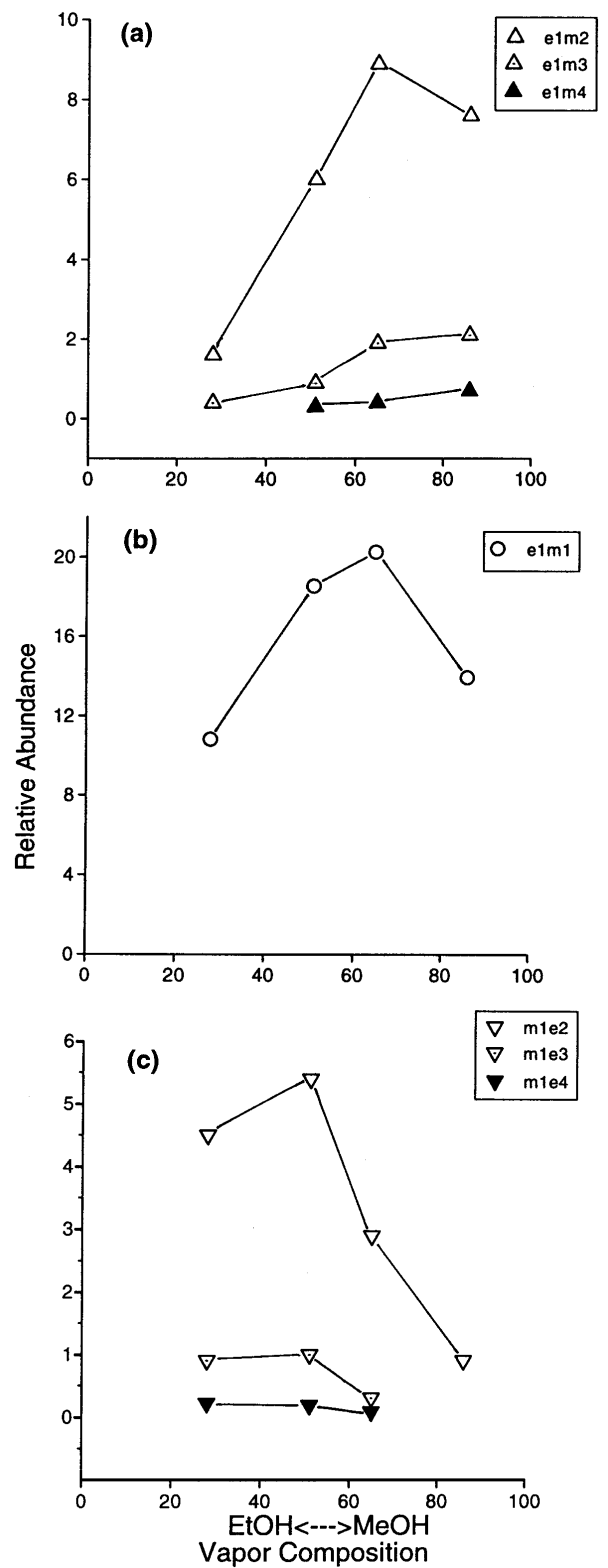

Figure 8. Variation of the intensities of the mass peaks corresponding to the hybrid clusters with the ratio of ethanol and methanol, (a) methanol-rich: up-triangles, (b) the hybrid dimer: circles, (c) ethanol-rich: down-triangles.

being investigated. A tunable dye laser will be added to the facility in the near future to carry out resonant ionization of clusters. 


\section{Acknowledgement}

The authors acknowledge the financial support of the Department of Science and Technology, Government of India under the instrumentation project. They thank Messrs V Sreenath and S Srinivas for technical assistance.

\section{References}

1. Schmid G 1994 Clusters and colloids, from theory to applications (Weinheim: VCH)

2. Edwards P P, Johnston R L and Rao C N R 1999 In Metal clusters in chemistry (eds) P Braunstein, G Oro, P R Raithby (New York: Wiley VCH)

3. Scoles G 1988 Atomic and molecular beam method vol 1 \& 2 (New York: Oxford University Press); Haberland H 1994 Clusters of atoms and molecules I \& II (Berlin: Springer-Verlag)

4. Ramsey N F 1948 Phys. Rev. 74286

5. Bentley P G 1961 Nature (London) 190432

6. Henkes W 1961 Z. Naturforsch. A16 842

7. Hopkins J B, Langridge-Smith P R R, Morse M D and Smalley R E 1983 J. Chem. Phys. 78 1627

8. Kroto H W, Heath J R, O'Brien S C, Curl R F and Smalley R E 1985 Nature (London) 318 162

9. Sakurai H and Castleman A W Jr 1997 J. Phys. Chem. A101 7695

10. Bililign S, Liu L, Feigerle C S and Miller J C 1997 J. Phys. Chem. A101 4569

11. Wei S and Castleman A W Jr 1994 Int. J. Mass Spectrom. Ion Process. 131233

12. Palmer P M and Topp M R 1998 Chem. Phys. Lett. 286113

13. Gruenloh C J, Florio G M, Carney J R, Hagemeister F C and Zwier T S 1999 J. Phys. Chem. A103 496

14. Jarrold M F and Creegan K M 1991 Chem. Phys. Lett. 166116

15. Wang L-S, Cheng H-S and Fan J 1995 J. Chem. Phys. 1029480

16. Heath J R, Liu Y, O’Brien S C, Zhang Q-L, Curl R F, Tittel F K and Smalley R E 1985 J. Chem. Phys. 835520

17. O'Brien S C, Liu Y, Zhang Q, Heath J R, Tittel F K, Curl R F and Smalley R E 1986 J. Chem. Phys. 844074

18. Barr D L 1987 J. Vac. Sci. Technol. B5 184

19. Cox D M, Trevor D J, Whetten R L, Rohlfing E A and Kaldor A 1985 Phys. Rev. B32 7290

20. Harfensit S A, Wang Z L, Whetten R L, Vezmar I and Alvarez M 1997 Adv. Mater. 9817

21. Sheuring T and Weil K 1985 Surf. Sci. 156457

22. Sattler K, Muhibach J and Recknagel E 1980 Phys. Rev. Lett. 45821

23. Jordan R M 1999 http://rmjordan.com/ttl.html

24. Maruyama S, Anderson L R and Smalley R E 1990 Rev. Sci. Instrum. 613686

25. Holden A 1965 The nature of solids (New York: Columbia University Press)

26. Bowers M T, Su T and Anicich V G 1973 J. Chem. Phys. 585175

27. Rohlfing E A, Cox D M and Kaldor A 1984 J. Chem. Phys. 813322

28. Campbell E E B, Ulmer G, Busmann H-G and Hertel I V 1990 Chem. Phys. Lett. 175505

29. Wegener P P and Wu B J C 1975 Faraday Discuss. Chem. Soc. 77

30. Wakisaka A, Abdoul-Carime H, Yamamoto Y and Kiyozumi Y 1998 J. Chem. Soc. Faraday Trans. 94369

31. Dahl D A 1995 SIMION 3D, Version 6.0, User's Manual Idaho National Engineering Laboratory, USA 